\title{
Care Prevention Needs in Community-Dwelling Older Adults in Japan
}

\author{
Ayumi Igarashi $^{*}{ }^{(D}$, Noriko Yamamoto-Mitani1, Akiko Ota ${ }^{2}$, \\ Tomoaki Ishibashi ${ }^{3}$, Naoki Ikegami ${ }^{4}$
}

\author{
${ }^{1}$ Department of Gerontological Home Care and Long-Term Care Nursing, Division of Health Sciences \& Nursing, Graduate \\ School of Medicine, The University of Tokyo, Tokyo, Japan \\ ${ }^{2}$ Brain Attack Center, Ota Memorial Hospital, Hiroshima, Japan \\ ${ }^{3}$ The Dia Foundation for Research on Ageing Societies, Tokyo, Japan \\ ${ }^{4}$ St. Luke's International University, Tokyo, Japan \\ Email: *aigarashi-tky@g.ecc.u-tokyo.ac.jp
}

How to cite this paper: Igarashi, A., Yamamoto-Mitani, N., Ota, A., Ishibashi, T. and Ikegami, N. (2021) Care Prevention Needs in Community-Dwelling Older Adults in Japan. Health, 13, 123-133.

https://doi.org/10.4236/health.2021.132011

Received: January 5, 2021

Accepted: February 15, 2021

Published: February 18, 2021

Copyright ( 2021 by author(s) and Scientific Research Publishing Inc. This work is licensed under the Creative Commons Attribution International License (CC BY 4.0).

http://creativecommons.org/licenses/by/4.0/ (c) (i) Open Access

\begin{abstract}
Care prevention comprises an integral part of long-term care in Japan and is provided across the following populations: those without problems when screened by the Basic Check List (BCL, Type 1), those having problems when screened by the BCL (Type 2), and those certified in the "support need levels" by the long-term care insurance eligibility assessment (Type 3 ). We aimed to clarify the care prevention needs across these three populations by using the interRAI Check-Up, an internationally developed instrument. We conducted cross-sectional surveys to assess care prevention needs for convenience samples of community-dwelling older adults in two cities in western Japan from October 2016 to December 2017, and we integrated the secondary data of older adults' assessment from September 2014 to June 2018. Prevalence rates of nine domains of care prevention needs were calculated. Among the 125 participants, 20 were Type 1, 23 were Type 2, and 82 were Type 3. All three types had the following needs that had not been assessed in the BCL: pains, risk of hospital-emergency room visits, driving reviews, and instrumental activities of daily living capacity. The results showed that interventions for a wide range of care prevention needs should be considered in the long-term care prevention project.
\end{abstract}

\section{Keywords}

Care Prevention, Community-Dwelling Older Adults, Comprehensive Assessment, Long-Term Care, Nursing 


\section{Introduction}

Increased age leads to physical/cognitive functional decline and declining health, with consequent high risk of falls, malnutrition, polypharmacy, and cognitive impairment [1]. Rapidly aging societies in developed countries are faced with the common challenge of preventing these risks in older adults and preserving their ability to independently carry out their daily activities. In Japan, the government introduced a long-term care (LTC) prevention project in the LTC insurance system in 2006 [2]. In the LTC prevention project, municipal governments have responsibilities for coordinating and providing care prevention services to community-dwelling older adults. The healthcare professionals, such as public health nurses/registered nurses, working for the community support center are responsible for supporting older adults.

The current LTC system in the country has three types of participants for the long-term care insurance (LTCI) prevention project: uncertified in the LTCI, healthy older adults (Type 1); uncertified in the LTCI, but certified as frail and at high risk for needing care by the Basic Check List (BCL, Type 2); and certified as "support need levels 1 or 2" in the LTCI (Type 3) [3]. Thus, there are two systems from which to select participants for LTC prevention: the LTCI certification system and the BCL. The certification system of the LTCI identifies older adults in "support need levels" of the LTCI system (Type 3); the BCL dichotomizes frail older adults (Type 2) from those who are healthy (Type 1). In the certification system of LTCI, the care managers designated by the municipality evaluate the physical and cognitive status of those being certified. To determine the certification level, the focus is on physical/cognitive functions such as movement/balance and conditions requiring assistance with activities of daily living and instrumental activities of daily living (ADL/IADL). Applicants are grouped into eligibility levels or as ineligible according to a designated algorithm developed for this purpose. In the BCL, on the other hand, the older adults or their family report the presence of problems for IADL, physical functioning, nutrition, eating, social interactions, memory, and depression [4].

Therefore, in the current LTC prevention project, municipalities independently apply two systems that have separate assessment items. Consequently, there is a discrepancy between the two processes by which the healthcare conditions are assessed to determine care prevention needs in the two systems. Furthermore, in these systems, standardized health assessment is not available for the risk of hospital admission or the emergency room (ER) visit, nor of the presence of pain.

The interRAI instrument is a standardized assessment tool for people who need assistance, such as older adults with disabilities, and it is used worldwide [5]. The interRAI Home Care (HC) is used by some provider agencies for the assessment of needs and care planning of older adults in the Japanese LTCI system. In previous studies, the interRAI instrument was used for comprehensive geriatric assessment, care planning/screening, and other measurements, and it 
had effects on improving functional outcomes, such as ADL, IADL, cognitive functions, and hospitalization/nursing home admission [6]. Among the interRAI series, the interRAI Check-Up (CU) has been focused on older adults living independently in the community and is composed of a subset of assessment items in the interRAI HC [7]. In the "COLLAGE program" in the USA, which aims to advance healthy aging and improve outcomes of community-dwelling older adults living independently, the interRAI CU has been used to identify an individual's health, functioning, and social needs and provide a health coaching program based on the assessment [8] [9]. A previous study revealed that a volunteer visit that was based on the assessment of interRAI CU improved self-reported well-being, emotional and informational support, social interaction, loneliness, depression, and levels of physical activity [10]. Thus, interRAI $\mathrm{CU}$ appears to be a useful tool for assessing the health conditions and care prevention needs of community-dwelling older adults.

We considered that interRAI CU might be helpful for a better assessment of the comprehensive care prevention needs of elderly people for the LTC prevention project in Japan. Therefore, we conducted a pilot survey to observe the prevalence of care prevention needs among the participants of the LTC prevention project using interRAI CU.

\section{Methods}

\subsection{Study Design}

This study was performed with a cross-sectional design using data from two data sources. The first data source was from a survey that had been conducted in two cities in western Japan (City A has a population of about 44,751, with a proportion of 38\% aged 65 and over in 2017; City B has a population of about 464,811, with a proportion of $26 \%$ aged 65 and over in 2015). The surveys were conducted from October 2016 to December 2017. The second data source was from a project conducted by the authors for measuring the quality of home care using the assessment data of interRAI HC. Data were collected from September 2014 to June 2018.

\subsection{Participants and Procedure}

Participants were convenience samples of community-dwelling older adults aged 65 years and older eligible for the LTC prevention project in Japan.

For the first survey, we recruited older adults who had not been certified by the LTCI in the community $(n=43)$, and those certified as having "support need levels" ( $n=35)$ from a care management agency, a small multifunctional group home (shokibo takinogata kyotaku kaigo in Japanese), and a day-care agency in the two cities. Investigators were nursing researchers or care managers in charge of care planning for the participants. For the recruitment, the nursing researchers asked the older adults who attended a group activity in the community to participate in this study; the care managers or nursing researchers asked those 
who used the care services in each agency during the survey period to participate. The investigators conducted assessments on the participants who agreed to participate using the interRAI CU. For those who had not been certified by the LTCI, the investigators also asked them to respond to the BCL for assessing frailty.

The second database was obtained from a project to evaluate quality by using quality indicators [11]. From this database, we extracted data for those who had been certified in the "support need levels" $(n=47)$. The assessments in the second survey were made by care managers in charge of care planning for the participants.

We classified the participants certified for care prevention into three types: those certified as healthy older adults (Type 1), those certified as frail (Type 2) by the BCL, and those certified as "support need levels" in the LTCI (Type 3) [3]. The screening by the BCL was based on the definition criteria in the guidelines published by the government (i.e., those who responded affirmatively to one or more of the following conditions: applying for multiple topics, declined locomotive functioning, malnutrition, declined oral functioning, staying indoors, decreased cognitive functioning, or possibly having depression) [12]. Those who did not respond affirmatively to any of the conditions were defined as "healthy" (Type 1). The older adults certified in the "support need levels" in the LTCI were defined as Type 3. Figure 1 shows the process for the recruitment and the classification of the participants.

\subsection{Measurements}

\subsubsection{InterRAI Check-Up and Care Prevention Needs}

To measure the functional and health conditions, we used the interRAI CU instrument [7], which had about 90 items and is a subset of the items in the interRAI HC. The assessment items consist of cognition/communication, mood/ psychosocial well-being, functional status, health conditions, disease diagnoses,

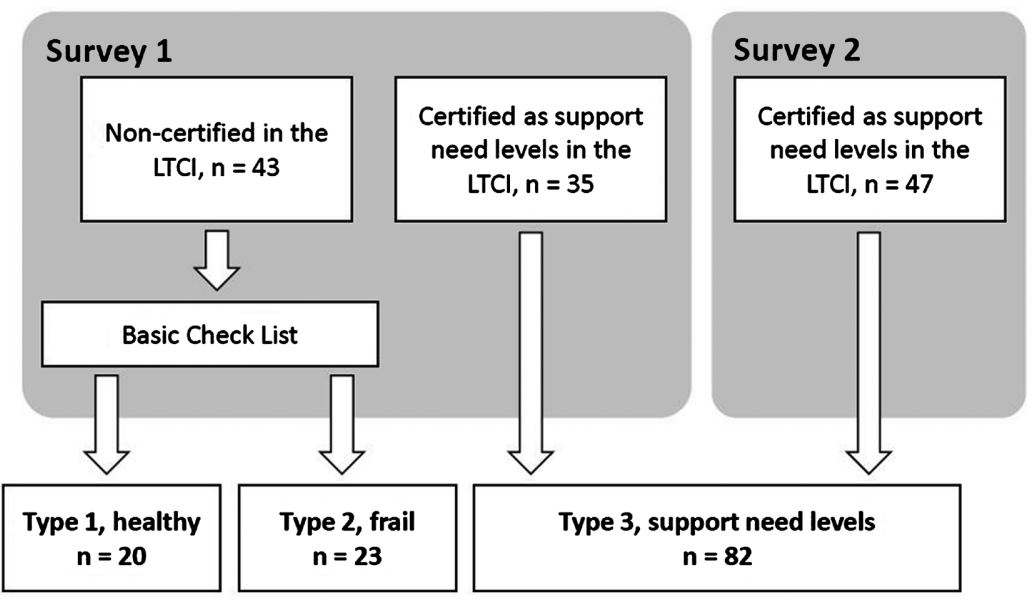

$\mathrm{LTCl}$ : long-term care insurance

Figure 1. Flow of recruitment and classification of participants. 
nutritional issues, procedures/treatments, and finances/stressors. The disease diagnosis was surveyed for coronary heart disease, chronic heart failure, chronic obstructive pulmonary disease, diabetes, cancer, cerebrovascular disease, and hypertension.

The investigators asked the participants about their functional and health conditions based on the assessment items of the interRAI CU.

Using the assessment data of the interRAI CU, we extracted nine care prevention needs: 1) falls, 2) moods (depressive symptoms), 3) cognition, 4) social activities, 5) driving review, 6) IADL, 7) hospital admission/ER visit, 8) nutrition, and 9) pain. We determined these needs for care prevention by referring to the algorithm of COLLAGE [9] and the scales calculated with interRAI instruments (Table 1).

\subsubsection{Participants' Characteristics}

We collected data on the demographic and health characteristics of the participants, including age, sex, and living arrangement, from the questionnaire for the interRAI assessment.

\subsubsection{Time Required for the Assessment}

To assess the feasibility of interRAI CU, we asked the investigators how much time they had spent on the assessment in the first survey.

\subsection{Ethical Considerations}

The investigators provided the participants with written documents describing the aims and procedures of the survey, the voluntary nature of participation, and that responses would be anonymous. We obtained written consent from the individuals who agreed to participate. The research ethics committee of the Graduate School of Medicine, The University of Tokyo, Tokyo, Japan, approved this study (No. 11461-(3)).

Table 1. Assessment items used for identifying needs based on interRAI CU.

\begin{tabular}{|c|c|}
\hline Needs & Scale/assessment items \\
\hline Falls & Prior fall, ADL status, unsteady gait, primary mode of locomotion, making self-understood (cognitive function), or dyspnea [9] \\
\hline Cognition & Score of 2 and above in Cognitive Performance Scale [14] \\
\hline Social activities & Reduced social interactions \\
\hline Nutrition & Weight loss of $5 \%$ or more in last 30 days, or $10 \%$ or more in last 180 days \\
\hline Driving review & Score of 3 and above in the Driving Scale [15] \\
\hline ADL capacity & $\begin{array}{l}\text { Daily decision making, change in decision making, primary mode of locomotion, change in ADL status, Alzheimer's disease, } \\
\text { dementia other than Alzheimer's disease [9] }\end{array}$ \\
\hline Hospital-ER visit & $\begin{array}{l}\text { Hospital visit, ER visit, daily decision making, dementia other than Alzheimer's disease, falls, dyspnea, lonely, unstable } \\
\text { conditions, capacity of managing medication, self-reported health, pain frequency [9] }\end{array}$ \\
\hline Pain & Score of 2 and above in Pain Scale [16] \\
\hline
\end{tabular}

Note. interRAI CU, interRAI Check-Up; ER, emergency room; ADL, activities of daily living. 


\subsection{Analysis}

We calculated descriptive statistics for the characteristics and the care prevention needs of the participants by the type of the LTC prevention project. SPSS version 22.0 was used for the analyses (IBM Corp., 2013).

\section{Results}

\subsection{Characteristics of Participants and Duration of Time for Assessment}

We analyzed data from a total of 125 participants (Type 1: $n=20$, Type 2: $n=$ 23, and Type 3: $n=82$ ). Table 2 shows the characteristics of the participants. The mean age was $72.8,73.6$, and 83.0 years in Types 1, 2, and 3, respectively; the proportion of females was $63.2 \%, 65.2 \%$, and $69.5 \%$ in Types 1,2 , and 3, respectively. While $95.0 \%$ of the participants in Type 1 and $82.6 \%$ of the participants in Type 2 lived with their family, $43.4 \%$ lived alone in Type 3. Altogether, $54.2 \%$, $27.3 \%$, and $19.7 \%$ of the participants had the diagnosis of hypertension, cerebrovascular disease, and diabetes, respectively.

The mean \pm standard deviation $(S D)$ duration of the time for the assessment using interRAI CU $(n=68)$ was $14.9 \pm 6.0$ minutes (range $=6$ - 35).

\subsection{Care Prevention Needs}

Table 3 shows the care prevention needs of the participants by the types of LTC prevention projects. Healthy older adult participants, categorized as Type $1(n=$ 20 ), had an average of $1.0 \pm 1.0$ (range $=0$ - 3) need; those who were frail, categorized as Type $2(n=23)$, had $1.6 \pm 1.6$ (range $=0-5)$ needs; those with the certification of support level needs in the LTC insurance, categorized as Type 3 $(\mathrm{n}=23)$, had $4.2 \pm 2.0$ (range $=1-8)$ needs.

Of the Type 1 participants, $35.0 \%$ had pain problems, and $35.0 \%$ had reduced social interactions. In the Type 2 participants, $43.5 \%$ had a fall risk, $30.4 \%$ had pain problems, $17.4 \%$ needed a driving review, $17.4 \%$ had reduced social interactions, and $17.4 \%$ had a risk of hospital admission/ER visit. In the Type 3 participants, $81.7 \%$ had a fall risk, $75.6 \%$ needed IADL capacity, $69.5 \%$ needed a driving review, $52.4 \%$ had cognitive decline, $39.0 \%$ had a risk of hospital admission/ER visit, $32.9 \%$ had pain problems, and $31.7 \%$ had reduced social interactions.

\section{Discussion}

In this study, we assessed the care prevention needs for community-dwelling older adults using the interRAI CU. To the best of our knowledge, this is the first study to clarify the care prevention needs across all participants of the LTC prevention project in Japan. Type 3 participants had significantly greater needs (with 4.2 needs on average) than Type 1 and 2 participants (1.0 and 1.6 needs, respectively), which corresponded to the certification levels in the LTC programs. All participants had prevention needs, which had not been assessed in the current system. 
Table 2. Characteristics of theparticipants.

\begin{tabular}{|c|c|c|c|c|c|c|c|c|}
\hline & \multicolumn{2}{|c|}{ Total $(n=125)$} & \multicolumn{2}{|c|}{ Type $1(n=20)$} & \multicolumn{2}{|c|}{ Type $2(n=23)$} & \multicolumn{2}{|c|}{ Type $3(n=82)$} \\
\hline & $n$ & (\%) & $n$ & (\%) & $n$ & (\%) & $n$ & (\%) \\
\hline \multicolumn{9}{|l|}{ City } \\
\hline City A & 56 & $(72.7)$ & 14 & $(70.0)$ & 14 & $(60.9)$ & 28 & $(82.4)$ \\
\hline City B & 21 & $(27.3)$ & 6 & $(30.0)$ & 9 & $(39.1)$ & 6 & $(17.6)$ \\
\hline Age, mean $\pm S D$ (range) & $80.0 \pm 8.7$ & $(65-101)$ & $72.8 \pm 6.8$ & $(65-88)$ & $73.6 \pm 6.0$ & $(65-84)$ & $83.0 \pm 7.9$ & $(65-101)$ \\
\hline \multicolumn{9}{|l|}{ Gender } \\
\hline Male & 40 & $(32.3)$ & 7 & $(36.8)$ & 8 & $(34.8)$ & 25 & $(30.5)$ \\
\hline Female & 84 & $(67.7)$ & 12 & $(63.2)$ & 15 & $(65.2)$ & 57 & $(69.5)$ \\
\hline \multicolumn{9}{|l|}{ Marital status } \\
\hline Never married & 3 & $(2.5)$ & 0 & $(0.0)$ & 2 & (8.7) & 1 & $(1.3)$ \\
\hline Married/partner & 57 & $(46.7)$ & 16 & $(80.0)$ & 14 & $(60.9)$ & 27 & $(34.2)$ \\
\hline Widowed & 56 & $(45.9)$ & 4 & $(20.0)$ & 6 & $(26.1)$ & 46 & $(58.2)$ \\
\hline Separated & 1 & $(0.8)$ & 0 & $(0.0)$ & 0 & $(0.0)$ & 1 & $(1.3)$ \\
\hline Divorced & 5 & $(4.1)$ & 0 & $(0.0)$ & 1 & $(4.3)$ & 4 & $(5.1)$ \\
\hline \multicolumn{9}{|l|}{ Living arrangement } \\
\hline Alone & 38 & $(31.9)$ & 1 & $(5.0)$ & 4 & $(17.4)$ & 33 & $(43.4)$ \\
\hline With spouse/partner only & 40 & $(33.6)$ & 11 & $(55.0)$ & 13 & $(56.5)$ & 16 & $(21.1)$ \\
\hline With spouse/partner and other(s) & 16 & $(13.4)$ & 5 & $(25.0)$ & 2 & (8.7) & 9 & $(11.8)$ \\
\hline With child (not spouse/partner) & 21 & $(17.6)$ & 3 & $(15.0)$ & 4 & $(17.4)$ & 14 & $(18.4)$ \\
\hline With parent(s) & 2 & (1.7) & 0 & $(0.0)$ & 0 & $(0.0)$ & 2 & $(2.6)$ \\
\hline With other relative(s) & 2 & $(1.7)$ & 0 & $(0.0)$ & 0 & $(0.0)$ & 2 & $(2.6)$ \\
\hline \multicolumn{9}{|l|}{ Education } \\
\hline Primary school & 4 & (3.9) & 1 & $(5.3)$ & 0 & $(0.0)$ & 3 & $(4.9)$ \\
\hline Junior high school & 23 & $(22.5)$ & 2 & $(10.5)$ & 6 & $(27.3)$ & 15 & $(24.6)$ \\
\hline High school & 55 & $(53.9)$ & 11 & $(57.9)$ & 10 & $(45.5)$ & 34 & $(55.7)$ \\
\hline Vocational school/junior college & 13 & $(12.7)$ & 3 & $(15.8)$ & 4 & $(18.1)$ & 6 & $(9.9)$ \\
\hline University & 7 & $(6.9)$ & 2 & $(10.5)$ & 2 & $(9.1)$ & 3 & $(4.9)$ \\
\hline \multicolumn{9}{|l|}{ Disease } \\
\hline Coronary artery disease & 15 & $(13.5)$ & 3 & $(18.8)$ & 2 & $(12.6)$ & 10 & $(12.6)$ \\
\hline Congested heart failure & 6 & $(5.4)$ & 0 & $(0.0)$ & 3 & $(17.6)$ & 3 & $(3.9)$ \\
\hline Chronic obstructive pulmonary disease & 3 & $(2.7)$ & 0 & $(0.0)$ & 0 & $(0.0)$ & 3 & (3.9) \\
\hline Diabetes & 23 & $(19.7)$ & 2 & $(11.8)$ & 5 & $(25.0)$ & 16 & $(20.3)$ \\
\hline Cancer & 13 & $(11.4)$ & 2 & $(11.8)$ & 2 & $(11.2)$ & 9 & $(11.4)$ \\
\hline Cerebrovascular disease & 30 & $(27.3)$ & 1 & $(6.3)$ & 4 & $(23.6)$ & 25 & $(32.5)$ \\
\hline Hypertension & 65 & $(54.2)$ & 6 & $(31.6)$ & 11 & $(52.4)$ & 48 & $(60.0)$ \\
\hline
\end{tabular}

Note. SD, standard deviation. 
Table 3. Care prevention needsby the types of LTC prevention projects.

\begin{tabular}{|c|c|c|c|c|c|c|c|c|}
\hline & \multicolumn{2}{|c|}{ Total $(n=125)$} & \multicolumn{2}{|c|}{ Type $1(n=20)$} & \multicolumn{2}{|c|}{ Type $2(n=23)$} & \multicolumn{2}{|c|}{ Type $3(n=82)$} \\
\hline & $n$ & (\%) & $n$ & (\%) & $n$ & (\%) & $n$ & (\%) \\
\hline Number of needs, mean $\pm S D$ (range) & $3.3 \pm 2.3$ & $(0-8)$ & $1.0 \pm 1.0$ & $(0-3)$ & $1.6 \pm 1.6$ & $(0-5)$ & $4.2 \pm 2.0$ & $(1-8)$ \\
\hline \multicolumn{9}{|l|}{ Needs } \\
\hline Falls & 79 & $(63.2)$ & 2 & $(10.0 \%)$ & 10 & $(43.5)$ & 67 & $(81.7)$ \\
\hline Moods & 16 & $(12.8)$ & 1 & $(5.0)$ & 1 & $(4.3)$ & 14 & $(17.1)$ \\
\hline Cognition & 47 & $(37.6)$ & 1 & $(5.0)$ & 3 & $(13.0)$ & 43 & $(52.4)$ \\
\hline Social activities & 37 & $(29.6)$ & 7 & $(35.0)$ & 4 & $(17.4)$ & 26 & $(31.7)$ \\
\hline Nutrition & 4 & $(3.2)$ & 0 & $(0.0)$ & 0 & $(0.0)$ & 4 & (4.9) \\
\hline Driving review & 61 & $(48.8)$ & 0 & $(0.0)$ & 4 & $(17.4)$ & 57 & $(69.5)$ \\
\hline IADL capacity & 66 & $(52.8)$ & 1 & $(5.0)$ & 3 & $(13.0)$ & 62 & $(75.6)$ \\
\hline Hospital-ER visit & 37 & $(29.6)$ & 1 & $(5.0)$ & 4 & $(17.4)$ & 32 & $(39.0)$ \\
\hline Pain & 41 & $(32.8)$ & 7 & $(35.0)$ & 7 & $(30.4)$ & 27 & (32.9) \\
\hline
\end{tabular}

Note. LTC, Long-term care; SD, standard deviation; IADL, instrumental activities of daily living; ER, emergency room.

Specifically, several participants had health-related needs, such as diagnosed disease(s) and needed pain management. Chronic diseases such as hypertension, cerebrovascular disease, and diabetes were frequent. The prevalence of those who had a risk of hospital admission/ER visit because of unstable health conditions gradually increased from Type 1 to Type 3 . The prevalence of pain problems was relatively high among all types (over 30\%). Management of pain, which is a common problem in older adults caused by joint or spine disorders, is essential for care prevention because it could lead to limiting activity [17], disabilities of physical functioning [18], and frailty [19] [20]. The results suggest that managing health conditions such as chronic diseases and pain symptoms should be performed from an early stage of the LTC prevention project.

Recently, the national government has attempted to promote the integration of health services and the LTC prevention project among community-dwelling older adults [21]. However, there are barriers to this initiative because the process of determining those who are in need is different depending on the measurement used. Moreover, disease management has not been given the attention required and little health-related information is available in the current LTC prevention programs. Health-related information obtained in the interRAI CU should be useful to assess the healthcare needs of older adults and would be able to serve as a bridge between the LTC services and healthcare services, thus contributing to improving health conditions and decreasing service needs. Public health nurses or registered nurses in community support centers can play a critical role in understanding and using health-related information effectively for care prevention.

Furthermore, the results revealed that Type 2 and 3 participants required 
driving reviews and had low levels of IADL capacity, which could be related to cognitive decline; these percentages increased from Type 2 to Type 3 participants. Although the BCL and the LTC certifications assess cognitive decline, strategies to address these problems have not been clarified. The need for a driving review and IADL capacity that surface from the interRAI CU assessment could provide a practical means to identify older persons needing care prevention services. While older adults aged 75 years or above must undergo an assessment of their cognitive function to renew their driving license under the Japanese Road Traffic Act, it is difficult to evaluate their driving aptitude based on the cognitive test alone [22]. By using the interRAI CU on a routine basis, information on aspects aside from cognitive decline, such as ADL, IADL, and vision, can be obtained. This would help physicians examine problems with older adults' driving ability.

Municipalities have been confronted with problems regarding how to manage the LTC prevention project efficiently and effectively. InterRAI CU would allow municipalities to gain a better understanding of the care prevention needs of all participants through an integrated database. The number of assessment items of the interRAI CU was reduced to one-third of the interRAI HC (full version). The time required to complete the assessment was 15 minutes on average, which suggests it is feasible for healthcare professionals to use the interRAI CU in their daily work. The self-reported form of the interRAI CU in an English version has recently been developed, and this may make the tool even more acceptable as the process could be made more efficient [7]. In the future, we plan to develop the Japanese version of the self-reported assessment form.

There were several limitations of this study. First, we recruited the participants using convenience sampling, and the sample size was relatively small because accessing potential participants through private healthcare agencies was difficult. Thus, the generalizability could not be confirmed. In future research, we aim to recruit community support centers managed by the municipality to assess the participants of the LTC prevention project comprehensively in the community. Second, it remains unclear which strategies are useful for preventing the need for care based on the needs extracted from the assessment. In the future, we would conduct longitudinal research to clarify the effects of the interventions based on the comprehensive assessment of care prevention.

\section{Conclusion}

In summary, this study experimentally applied a new assessment tool, interRAI $\mathrm{CU}$, for the participants of the LTC prevention project in Japan and identified the care prevention needs that are not assessed in the current LTC system. InterRAI CU may be useful for the integrated management of the LTC prevention project. Further, care prevention provided by nurses in the community support centers may be more effective using a standardized and comprehensive assessment tool such as interRAI CU. 


\section{Acknowledgements}

We thank all the participants and investigators.

\section{Conflicts of Interest}

The authors declare no conflicts of interest regarding the publication of this paper.

\section{References}

[1] Cronfalk, B.S., Fjell, A., Carstens, N., Rosseland, L.M.K., Rongve, A., Rönnevik, D.H., et al. (2017) Health Team for the Elderly: A Feasibility Study for Preventive Home Visits. Primary Health Care Research \& Development, 18, 242-252. https://doi.org/10.1017/S1463423617000019

[2] Fukutomi, E., Kimura, Y., Wada, T., Okumiya, K. and Matsubayashi, K. (2013) Long-Term Care Prevention Project in Japan. The Lancet, 381, 116. https://doi.org/10.1016/S0140-6736(13)60049-5

[3] Tsutsui, T. and Muramatsu, N. (2007) Japan's Universal Long-Term Care System Reform of 2005: Containing Costs and Realizing a Vision. Journal of the American Geriatrics Society, 55, 1458-1463. https://doi.org/10.1111/j.1532-5415.2007.01281.x

[4] Kera, T., Kawai, H., Yoshida, H., Hirano, H., Kojima, M., Fujiwara, Y., et al. (2017) Classification of Frailty Using the Kihon Checklist: A Cluster Analysis of Older Adults in Urban Areas. Geriatrics \& Gerontology International, 17, 69-77. https://doi.org/10.1111/ggi.12676

[5] Hirdes, J.P., Ljunggren, G., Morris, J.N., Frijters, D.H.M., Soveri, H.M., Gray, L., et al. (2008) Reliability of the interRAI Suite of Assessment Instruments: A 12-Country Study of an Integrated Health Information System. BMC Health Services Research, 8 , 277. https://doi.org/10.1186/1472-6963-8-277

[6] Salahudeen, M.S. and Nishtala, P.S. (2019) A Systematic Review Evaluating the Use of the interRAI Home Care Instrument in Research for Older People. Clinical Gerontologist, 42, 463-484. https://doi.org/10.1080/07317115.2018.1447525

[7] Morris, J.N., Howard, E.P., Geffen, L.N., Hirdes, J.P. and Hogeveen, S. (2018) InterRAI Check-Up (CU) Assessment, Supplement, and Self-Reported Forms and User's Manual, Version 10. InterRAI, Washington DC.

[8] Howard, E.P. and Louvar, K.E. (2017) Examining Life Goals of Community-Dwelling, Low-Income Older Adults. Research in Gerontological Nursing, 10 , 205-214. https://doi.org/10.3928/19404921-20170831-02

[9] Howard, E.P., Schreiber, R., Morris, J.N., Russotto, A. and Flashner-Fineman, S. (2016) COLLAGE 360: A Model of Person-Centered Care to Promote Health among Older Adults. Journal of Ageing Research and Healthcare, 1, 21-30. https://doi.org/10.14302/issn.2474-7785.jarh-16-1123

[10] Geffen, L.N., Kelly, G., Morris, J.N. and Howard, E.P. (2019) Peer-to-Peer Support Model to Improve Quality of Life among Highly vulnerable, Low-Income Older Adults in Cape Town, South Africa. BMC Geriatrics, 19, 279.

https://doi.org/10.1186/s12877-019-1310-0

[11] Ikegami, N., Ishibashi, T. and Amano, T. (2014) Japan’s Long-Term Care Regulations Focused on Structure-Rationale and Future Prospects. In: Mor, V., Leone, T. and Maresso, A., Eds., Regulating Long-Term Care Quality. An International Comparison, Cambridge University Press, New York, 121-143. https://doi.org/10.1017/CBO9781107323711.008 
[12] Ministry of Health, Labour and Welfare (2018) Guideline of the Integrated Project for Care Prevention and Daily Life Support 2018.

https://www.mhlw.go.jp/file/06-Seisakujouhou-12300000-Roukenkyoku/000008827 6.pdf

[13] Burrows, A.B., Morris, J.N., Simon, S.E., Hirdes, J.P. and Phillips, C. (2000) Development of a Minimum Data Set-Based Depression Rating Scale for Use in Nursing Homes. Age and Ageing, 29, 165-172. https://doi.org/10.1093/ageing/29.2.165

[14] Morris, J.N., Fries, B.E., Mehr, D.R., Hawes, C., Phillips, C., Mor, V., et al. (1994) MDS Cognitive Performance Scale. Journal of Gerontology, 49, M174-M182. https://doi.org/10.1093/geronj/49.4.M174

[15] Morris, J.N., Howard, E.P., Fries, B.E., Berkowitz, R., Goldman, B. and David, D. (2014) Using the Community Health Assessment to Screen for Continued Driving. Accident Analysis \& Prevention, 63, 104-110. https://doi.org/10.1016/j.aap.2013.10.030

[16] Fries, B.E., Simon, S.E., Morris, J.N., Flodstrom, C. and Bookstein, F.L. (2001) Pain in U.S. Nursing Homes: Validating a Pain Scale for the Minimum Data Set. The Gerontologist, 41, 173-179. https://doi.org/10.1093/geront/41.2.173

[17] Monma, T., Takeda, F., Noguchi, H. and Tamiya, N. (2016) Age and Sex Differences of Risk Factors of Activity Limitations in Japanese Older Adults. Geriatriatrics \& Gerontology International, 16, 670-678. https://doi.org/10.1111/ggi.12533

[18] Makino, K., Lee, S., Bae, S., Jung, S., Shinkai, Y., Chiba, I., et al. (2019) Pain Characteristics and Incidence of Functional Disability among Community-Dwelling Older Adults. PLoS ONE, 14, e0215467. https://doi.org/10.1371/journal.pone.0215467

[19] Nakai, Y., Makizako, H., Kiyama, R., Tomioka, K., Taniguchi, Y., Kubozono, T., et al. (2019) Association between Chronic Pain and Physical Frailty in Community-Dwelling Older Adults. International Journal of Environmental Research and Public Health, 16, 1330. https://doi.org/10.3390/ijerph16081330

[20] Coelho, T., Paúl, C., Gobbens, R.J.J. and Fernandes, L. (2017) Multidimensional Frailty and Pain in Community Dwelling Elderly. Pain Medicine, 18, 693-701. https://doi.org/10.1111/pme.12746

[21] Ministry of Health, Labour and Welfare (2019) Report of the Review Group for the Program for Promoting the Integrated Implementation of Health Services and Care Prevention for Older Adults. https://www.mhlw.go.jp/content/12401000/000551951.pdf

[22] Kamimura, N. (2018) Impacts on Clinical and Life in Driving of People with Dementia. Gerontopsychiatry, 29, 825-833. 